\title{
Burden of Depression among Working-Age Adults with Rheumatoid Arthritis
}

\author{
Arijita Deb (D), ${ }^{1}$ Nilanjana Dwibedi, ${ }^{1}$ Traci LeMasters, ${ }^{1}$ Jo Ann Hornsby, ${ }^{2}$ \\ Wenhui Wei, ${ }^{3}$ and Usha Sambamoorthi ${ }^{1}{ }^{1}$ \\ ${ }^{1}$ School of Pharmacy, Department of Pharmaceutical Systems and Policy, West Virginia University, Morgantown, WV, USA \\ ${ }^{2}$ School of Medicine, West Virginia University, Morgantown, WV, USA \\ ${ }^{3}$ Regeneron Pharmaceuticals, Tarrytown, NJ, USA
}

Correspondence should be addressed to Arijita Deb; ardeb@mix.wvu.edu

Received 6 December 2017; Accepted 8 April 2018; Published 3 June 2018

Academic Editor: Changhai Ding

Copyright (C) 2018 Arijita Deb et al. This is an open access article distributed under the Creative Commons Attribution License, which permits unrestricted use, distribution, and reproduction in any medium, provided the original work is properly cited.

Objective. This study estimated the excess clinical, humanistic, and economic burden associated with depression among workingage adults with Rheumatoid Arthritis (RA). Methods. A retrospective cross-sectional study was conducted among working-age (18 to 64 years) RA patients with depression $(N=647)$ and without depression $(N=2,015)$ using data from the nationally representative Medical Expenditure Panel Survey for the years 2009, 2011, 2013, and 2015. Results. Overall, 25.8\% had depression. In adjusted analyses, adults with RA and depression compared to those without depression were significantly more likely to have pain interference with normal work (severe pain: $\mathrm{AOR}=2.22 ; 95 \% \mathrm{CI}=1.55,3.18$ ), functional limitations $(\mathrm{AOR}=2.17 ; 95 \% \mathrm{CI}$ $=1.61,2.94)$, and lower mental health HRQoL scores. Adults with RA and depression had significantly higher annual healthcare expenditures $(\$ 14,752$ versus $10,541, p<.001)$ and out-of-pocket spending burden. Adults with RA and depression were more likely to be unemployed and among employed adults, those with depression had a significantly higher number of missed work days annually and higher lost annual wages due to missed work days. Conclusions. This study highlights the importance of effectively managing depression in routine clinical practice of RA patients to reduce pain and functional limitations, improve quality of life, and lower direct and indirect healthcare costs.

\section{Introduction}

Rheumatoid Arthritis (RA) is one of the most debilitating chronic conditions, with the onset often occurring during the prime working years of lives, between the ages of 20 and 40 years [1]. Individuals with RA experience substantial pain and RA causes permanent work disability in more than one-third of affected patients within 10 years of onset [2]. Such pain and disabilities associated with RA may contribute to a higher prevalence of depression in individuals with RA compared to healthy controls [3]. An expert review of depression in arthritis reported that the prevalence of depression in adults with RA can be as high as $66.2 \%$ [4]. A systematic review and meta-analysis of 72 studies estimated the prevalence rate to be $16.8 \%$ [5].

The disease burden of depression in RA can be substantial because depression can worsen survival [6] and increase morbidity in terms of disability, health-related quality of life (HRQoL), RA disease activity [7], and pain [8]. Depression in RA can also increase healthcare resource utilization $[9,10]$, which can lead to high health care expenditures for both insurance payers, patients and families. As depression in RA can aggravate disability, an individual may also suffer economic losses due to work inability or even experience economic losses due to missed work days. Although not specific to RA, one study estimated that 6.9 million workingage adults reported arthritis-attributable work limitation [11]. One can speculate that depression can worsen the work limitation because the presence of depression along with any chronic physical condition more than doubles the likelihood of work absenteeism as compared to the presence of any chronic physical condition without depression [12].

However, to date, no published study in the US has done a comprehensive analysis of the humanistic and economic 


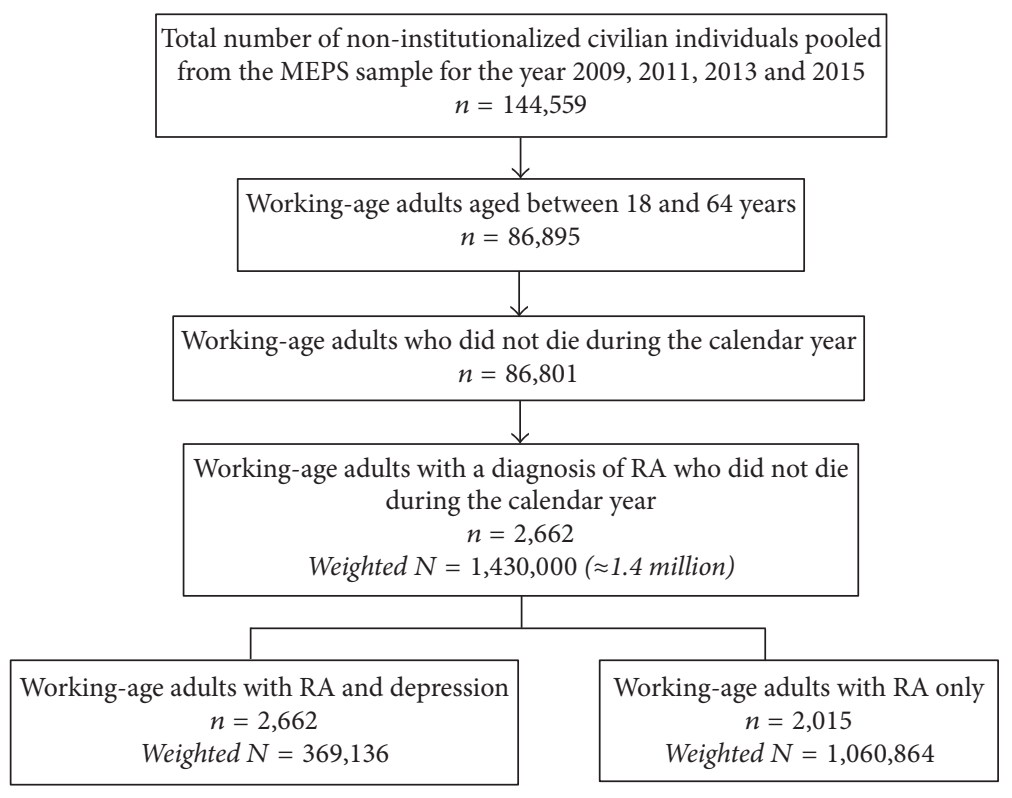

FIgURE 1: Flow diagram of study sample.

burden associated with depression among RA patients, particularly among working-age adults. In fact, a recent review highlighted the significant knowledge gap in estimating the disease burden of depression in adults with arthritis [4]. Although there has been a handful of studies on the association between depression and HRQoL among adults with RA, most of these studies have been conducted outside the US $[13,14]$ or only among women in a specific setting [15]. One US study used a cross-sectional design and examined the relationship between depression and disability, HRQoL in the US with data from the 2011 Behavioral Risk Factor Surveillance System [16]. However, this study included all forms of arthritis and did not focus on RA. Again, only one study using 2006 commercial claims data in the US found that RA patients with depression had a significantly higher adjusted annual healthcare costs as compared to RA patients without depression $(\$ 12,225$ versus $\$ 11,404)$ [9]. However, this study was based on commercially insured RA patients and may not be representative of the US national population. Furthermore, this commercial insurance data did not include certain patient characteristics which are important confounders of healthcare costs such as race, education, and income level.

Therefore, the objective of this study is to examine the incremental burden of depression on the clinical, humanistic, and economic outcomes among working-age adults with RA.

\section{Methods}

2.1. Study Design. A retrospective cross-sectional study design with data from a nationally representative sample of working-age adults (18-64 years) was used.

2.2. Data Source. We used data from the Medical Expenditure Panel Survey (MEPS), an annual household survey of the noninstitutionalized civilian population in the US. Information on demographic characteristics, medical conditions, health status, utilization of health care services, charges and payments, access to care, health insurance coverage, income, education, employment, and missed workdays of the participants in the survey were extracted from the household component of MEPS. We pooled four years of data (2009, 2011, 2013, and 2015) to have sufficient sample size and used data from alternate years to avoid including two observations per individual. Furthermore, a question related to the type of arthritis was available in these years. MEPS recommends pooling of data to increase sample size and it is a common practice in published literature with MEPS data [17].

2.3. Study Sample. The study sample $(N=2,662)$ consisted of working-age (18-64 years) who were diagnosed with RA and who were alive during the study period (2009, 2011, 2013, and 2015) (Figure 1). RA was identified from the priority condition enumeration section. In this section, respondents were queried whether an individual in the household has ever been told by a doctor or another health professional that she/he had arthritis and type of arthritis (RA versus osteoarthritis). We also identified RA from medical condition file with the clinical classification code (202). Medical conditions were reported by the respondents if they sought treatment for the condition, or if the condition resulted in disability, or if the condition was bothersome. The responses were recorded as texts, and these texts were translated into International Classification of Diseases, 9th Edition, Clinical Modification (ICD-9-CM) codes by professional coders. In addition, MEPS data provides clinical classification codes, which are aggregated ICD-9-CM codes into clinically meaningful categories that group similar conditions (Agency for Healthcare Research and Quality). 
2.4. Conceptual Framework. The conceptual framework for this study was adapted from the Andersen's Expanded Behavioral Model which posits that health services utilization and outcomes of an individual are a function of predisposing factors (e.g., age, sex, and race), enabling factors (e.g., marital status, education and poverty status), need factors (e.g., chronic conditions, health status), and personal health practices (e.g., physical activity, obesity, and smoking) [18].

\subsection{Measures}

\subsubsection{Clinical Outcomes}

Pain Interference with Normal Activities. Based on a selfadministered single-item question, pain interference with normal activities during the past four weeks among the household respondents was measured. The answers were recorded on a 5-point Likert scale during the past 4 weeks. In MEPS, pain was reported on a 5-point scale: (1) not at all, (2) a little bit, (3) moderately, (4) quite a bit, and (5) extremely. For purposes of this study we group pain categories as follows: (1) not at all/little bit; (2) moderate; (3) severe (quite a bit/extremely). Self-reported pain from MEPS has been used in published literature to estimate the cost of pain [19]. There were 42 individuals (5.9\%) in the depression group and 140 individuals $(6.6 \%)$ in the no depression group with missing data on pain inference variable. These individuals were not included in the analysis.

Any Functional Limitations. This variable summarizes whether an individual had any limitations in instrumental activities of daily living (IADL) (e.g., shopping, cooking, using phone, paying bills, taking medications, driving, doing laundry, or going shopping), activities of daily living (ADL) (e.g., bathing, dressing, grooming, mouthcare, toileting, and eating), functional limitations (walking, climbing stairs, grasping objects, reaching overhead, lifting, bending or stooping, or standing for long periods of time), or activity limitations (work, housework, or school).

2.5.2. Humanistic Outcome: Health-Related Quality of Life. HRQoL was measured by the generic Short-Form-12 Version 2 (SF12-V2) summary scores. The SF12-V2 is a generic multipurpose survey with 12 questions, which encompass eight domains (role physical, role emotional, physical function, social function, mental health, vitality, pain, and general health). These questions are designed to provide summary measures of overall HRQoL of an individual. The Mental Component Summary (MCS) score was derived from the responses to the items in the domains: vitality, social functioning, role emotional (limitations in work and daily activities because of emotional problems), and mental health. The Physical Component Summary (PCS) score was derived from the responses to the items in the domains: physical functioning, role physical, bodily pain, and general health. Both MCS and PCS scores ranged from 0 to 100, with higher scores representing better self-reported health and better HRQoL related to mental or physical health [20].

\subsubsection{Economic Outcomes: Direct Healthcare Expenditures}

Total Healthcare Expenditures. In the MEPS, expenditures are defined as the sum of direct payments for care provided during the year. The direct payments include twelve sources of payment categories such as out-of-pocket by patient or families, Medicare, Medicaid, Private Insurance, Veteran Administration, worker's compensation, and others. Total annual per person healthcare expenditures were calculated as the sum of inpatient, outpatient, emergency, dental, home health, vision, prescription drugs, and other medical supplies. All expenditures were inflation adjusted to 2015 US dollars (USD) using consumer price index for medical services from the bureau of medical services.

Total Out-of-Pocket Spending Burden by Patients and Families. We also estimated the total out-of-pocket spending on healthcare by the respondent and/or family. These included annual deductibles, copayment, and coinsurance for services and payment for services that were not covered by health insurance. We calculated out-of-pocket spending burden as the ratio of out-of-pocket healthcare expenditures to personal income [21], which varied from zero to 100. Based on published literature, we defined spending $10 \%$ or more of personal income on health care as high out-of-pocket spending burden [22].

\subsubsection{Economic Outcomes: Indirect Healthcare Burden}

Unemployment (i.e., Labor Market Outcome). In the MEPS, employment section covers questions about each person's employment or self-employment status. Based on these questions, we classified individuals who were currently unemployed.

Missed work days were measured whether individuals lost a half-day or more from work because of illness, injury, or mental or emotional problems during the year and how many workdays were lost. This was calculated only for employed adults.

Lost wages for each individual were calculated by multiplying missed work days with an average daily wage of each individual. All wages were adjusted to 2015 general consumer inflation rates derived from the bureau of labor statistics.

\section{Key Explanatory Variable}

Depression (Yes/No). Depression was identified based on the clinical classification code "657," which included both depressive disorders and bipolar disorders.

Other Explanatory Variables. Predisposing characteristics were sex (male, female), race/ethnicity (Whites, AfricanAmerican, and other racial minorities), and age in years (18-39, 40-49, and 50-64). Enabling factors comprised marital status (married, widow, separated/divorced, and never married), family poverty status (not poor, poor), health insurance status (public, private), and usual source of care (yes, no). Need factors included having a chronic condition other than RA from a list of eight conditions (asthma, 
cancer, chronic obstructive pulmonary disease, diabetes, heart disease, hypertension, stroke, and thyroid), anxiety, perceived physical health status (excellent/very good, good, and fair/poor), and perceived mental health status (excellent/very good, good and fair/poor). Personal health practice factors included obesity (obese and not obese), smoking status (current smoker, others, and missing), and exercise ("yes" and "no" exercise).

2.6. Statistical Analyses. A variety of statistical analyses were used based on the measurement of the outcome variables. The unadjusted relationships between the presence of depression and categorical variables and outcomes (pain interference with activities, employment, and OOP burden) were assessed with chi-square tests. Unadjusted differences in continuous outcomes (PCS, MCS, all-cause healthcare expenditures, and out-of-pocket spending by the patients and their families) by depression were tested with $t$-tests. Multinomial logistic regression was used to analyze the association between depression and pain-related interference with normal work after adjusting for the predisposing, enabling, need, and external environment characteristics. Logistic regression was used to analyze the association between depression and binary categorical variables (e.g., any limitations, unemployment, and out-of-pocket spending burden) after adjusting for covariates. Adjusted models for continuous outcomes (expenditures, out-of-pocket expenditures, and lost wages) consisted of Generalized Linear Models (GLM). GLM is flexible and can handle categorical outcomes, continuous outcomes, and count-data with the appropriate distribution family and a link function. For count-data (e.g., the number of missed work days) we used negative binomial regression.

Counterfactual Prediction Technique (Recycled Prediction). We used counterfactual recycled prediction, an approach that is gaining attention $[23,24]$ to estimate excess total healthcare expenditures, prescription expenditures, missed work days, and lost wages attributable to depression among working-age adults with Rheumatoid Arthritis. The recycled prediction technique is a preferred approach because it adjusts for differences in characteristics between the depression and no depression group by creating counterfactual scenarios. In all recycled prediction models, confidence intervals were obtained using 2000 bootstrap replications using the percentile method. To account for the complex design of MEPS, we conducted all analyses using the survey procedures in Statistical Analysis Software (SAS) version 9.3, Cary, NC, USA, and the survey design features with STATA 14. As we pooled four years, to get annualized weighted numbers, we divided the weights by four, recommended by the MEPS investigators [25] and used in the published literature [26].

\section{Results}

3.1. Description of the Study Sample. Majority of the study sample was female (64\%) and white (63\%), aged between 50 and 64 years (61\%), and had multimorbidity (72\%). Only $26 \%$ of individuals who perceived themselves having excellent or very good physical health and $44.8 \%$ reported having excellent or very good mental health (see Table 1).

Overall, $25.8 \%$ of adults with RA reported depression (Table 2). We observed significant differences in the rate of depression by predisposing, enabling, need factors, and personal health practices except for age, education, and region. For example, female adults with RA reported a significantly higher rate of depression than their male counterparts $(29.6 \%$ versus $19.1 \%)$. The higher rate of depression was also observed among individuals with multimorbidity (29.1\% versus $17.4 \%)$. A higher percentage of those who perceived themselves to be poor/fair physical health reported depression compared to those in excellent or very good health (38.0\% versus $12.4 \%)$.

\subsection{Clinical Outcomes}

3.2.1. Pain Interference with Normal Activities. A higher percentage of adults with RA and depression reported severe pain interfering with work or other normal activities compared to those with RA and no depression (54.8\% versus $30.8 \%$ ) (Table 3). After adjusting for predisposing, enabling, need, personal health practices, and external environment factors, and adults with depression were 2.2 times as likely to report severe pain interference with normal work activities than those without depression $(\mathrm{AOR}=2.22 ; 95 \% \mathrm{CI}=1.55$, 3.18) (Table 3).

3.2.2. Any Functional Limitations. A significantly higher percentage of adults with RA and depression reported any functional limitations compared to those with RA and no depression (79\% versus 51.1\%) (Table 3). After adjusting for covariates adults with RA and depression were more than 2 times as likely to report any functional limitations $(\mathrm{AOR}=$ 2.24; $95 \% \mathrm{CI}=1.62,3.10$ ) (Table 3$)$.

3.3. Humanistic Outcomes. Adults with RA and depression reported significantly lower HRQoL scores in both Physical Component Summary score (35.1 versus 40.2, $p<.001$ ) and Mental Component Summary score (37.2 versus 48.7, $p<.001)$ compared to adults with RA without depression (Table 4). In adjusted analyses, a significant difference was observed only in the mental domain of the HRQoL; the presence of depression was associated with a decrement of 8.72 in MCS scores (Table 4). The counterfactual predictions yielded similar differences in MCS (37.19 in adults with depression versus 45.91 in adults without depression, $p<$ $.001)$. The relationship between depression and PCS scores became insignificant after adjustment for the presence of multiple chronic conditions.

\subsection{Economic Outcomes}

3.4.1. Direct Total Healthcare Expenditures. In unadjusted analysis, adults with RA and depression had significantly higher annual healthcare expenditures (\$17,941 versus $\$ 10,064 p<.001)$. In the adjusted GLM with gamma distribution and log-link, we found that depression was associated with greater total healthcare expenditures compared to those without depression (Beta $=0.34, \mathrm{SE}=0.08)$. When converted 
TABLE 1: Description of study sample. Working-age (18 to 64 years) adults with Rheumatoid Arthritis Medical Expenditure Panel Survey (2009, 2011, 2013, and 2015).

\begin{tabular}{|c|c|c|c|}
\hline & $N$ & Weighted $N$ & Weighted\% \\
\hline All & 2,662 & $5,719,998$ & 100.0 \\
\hline \multicolumn{4}{|l|}{ Gender } \\
\hline Female & 1,826 & $3,661,958$ & 64.0 \\
\hline Male & 836 & $2,058,040$ & 36.0 \\
\hline \multicolumn{4}{|l|}{ Race/ethnicity } \\
\hline White & 1,061 & $3,596,249$ & 62.9 \\
\hline African American & 778 & 989,822 & 17.3 \\
\hline Latino & 639 & 780,416 & 13.6 \\
\hline Others & 184 & 353,511 & 6.2 \\
\hline \multicolumn{4}{|l|}{ Age in years } \\
\hline $18-39$ years & 444 & 956,652 & 16.7 \\
\hline $40-49$ years & 609 & $1,271,670$ & 22.2 \\
\hline $50-59$ years & 1,080 & $2,326,292$ & 40.7 \\
\hline 60-64 years & 529 & $1,165,384$ & 20.4 \\
\hline \multicolumn{4}{|l|}{ Marital status } \\
\hline Married & 1,287 & $3,098,420$ & 54.2 \\
\hline Widow/separated/divorced & 845 & $1,630,180$ & 28.5 \\
\hline Never married & 530 & 991,398 & 17.3 \\
\hline \multicolumn{4}{|l|}{ Education } \\
\hline Less than high school & 665 & $1,007,827$ & 17.6 \\
\hline High school & 939 & $2,043,963$ & 35.7 \\
\hline More than high school & 1,036 & $2,623,400$ & 45.9 \\
\hline \multicolumn{4}{|l|}{ Poverty status } \\
\hline Poor & 740 & $1,216,767$ & 21.3 \\
\hline Not poor & 675 & $1,204,168$ & 21.1 \\
\hline Middle income & 708 & $1,670,010$ & 29.2 \\
\hline High income & 539 & $1,629,054$ & 28.5 \\
\hline \multicolumn{4}{|l|}{ Insurance status } \\
\hline Private & 1,302 & $3,411,533$ & 59.6 \\
\hline Public & 932 & $1,580,322$ & 27.6 \\
\hline Uninsured & 428 & 728,144 & 12.7 \\
\hline \multicolumn{4}{|l|}{ Employment } \\
\hline Employed & 1,309 & $3,071,100$ & 53.7 \\
\hline Not employed & 1,352 & $2,647,283$ & 46.3 \\
\hline \multicolumn{4}{|l|}{ Region } \\
\hline Northeast & 383 & 984,779 & 17.2 \\
\hline Midwest & 523 & $1,264,228$ & 22.1 \\
\hline South & 1,190 & $2,454,395$ & 42.9 \\
\hline West & 566 & $1,016,597$ & 17.8 \\
\hline \multicolumn{4}{|l|}{ Perceived physical health } \\
\hline Excellent/very good & 607 & $1,488,614$ & 26.0 \\
\hline Good & 871 & $2,001,187$ & 35.0 \\
\hline Fair/poor & 1,184 & $2,230,197$ & 39.0 \\
\hline \multicolumn{4}{|l|}{ Perceived mental health } \\
\hline Excellent/very good & 607 & $1,488,614$ & 26.0 \\
\hline Good & 871 & $2,001,187$ & 35.0 \\
\hline Fair/poor & 1184 & $2,230,197$ & 39.0 \\
\hline
\end{tabular}

TABLE 1: Continued.

\begin{tabular}{lccc}
\hline & $N$ & Weighted $N$ & Weighted $\%$ \\
\hline Multimorbidity & & & \\
$\quad$ RA only & 716 & $1,605,886$ & 28.1 \\
$\quad$ Multimorbidity & 1,946 & $4,114,112$ & 71.9 \\
Anxiety & & & \\
$\quad$ Yes & 494 & $1,134,341$ & 19.8 \\
$\quad$ No & 2,168 & $4,585,657$ & 80.2 \\
Obesity & & & \\
$\quad$ Obese & 1,218 & $2,521,340$ & 44.1 \\
$\quad$ Not obese & 1,392 & $3,084,702$ & 53.9 \\
Smoking status & & & \\
Current smoker & 698 & $1,567,457$ & 27.4 \\
$\quad$ Others & 1,755 & $3,729,925$ & 65.2 \\
$\quad$ Missing & 209 & 422,616 & 7.4 \\
Exercise & & & \\
$\quad$ Yes & 1,077 & $2,364,802$ & 41.3 \\
No & 1,568 & $3,321,567$ & 58.1 \\
\hline
\end{tabular}

Note. Based on 2,662 adults with Rheumatoid Arthritis, aged between 18 and 64 years, who were alive during the calendar year. Missing data for the variables, education, obesity, smoking, and exercise, are not presented. Weighted $N$ and percentages were derived by dividing the person weights by the number of years pooled.

to original dollars this represented $\$ 14,752$ for those with depression and $\$ 10,541$ for those without depression (Table 5). Estimates from counter-factual recycled prediction revealed that depression was associated with an excess of $\$ 4,212$ total healthcare expenditures with $95 \% \mathrm{CI}=\$ 4,114$, $\$ 4,318$.

In unadjusted analysis, patients/families in the RA + depression group spent significantly higher amounts out-ofpocket on health care compared to the RA + no depression group ( $\$ 1,443$ versus $\$ 1,052, p<.001)$. In the adjusted GLM with gamma distribution and log-link, we found that depression was associated with greater total out-of-pocket healthcare spending compared to those without depression (Beta $=0.23, \mathrm{SE}=0.06)$. When converted to original dollars this represented \$1,232 for those with depression and $\$ 979$ for those without depression (Table 5). Estimates from counterfactual recycled prediction revealed that depression was associated with an excess of $\$ 253$ with $95 \% \mathrm{CI}=\$ 247,260$.

When high out-of-pocket spending burden was measured as spending greater than $10 \%$ of income on healthcare, we found that $30.7 \%$ of adults with depression and $21.3 \%$ of adults without depression had high out-of-pocket spending burden. After adjusting for other factors, adults with depression were significantly more likely to have high out-of-pocket spending burden $(\mathrm{AOR}=1.34 ; 95 \% \mathrm{CI}=1.01,1.79)$.

\subsubsection{Indirect Economic Burden}

Labor Market Outcome (Unemployment), Missed Work Days, and Lost Wages. Presence of depression was significantly associated with unemployment among adults with RA; $64.1 \%$ of adults with depression were unemployed compared to 
TABLE 2: Description of study sample by depression among working-age (18-64 years) adults with Rheumatoid Arthritis Medical Expenditure Panel Survey (2009, 2011, 2013, and 2015).

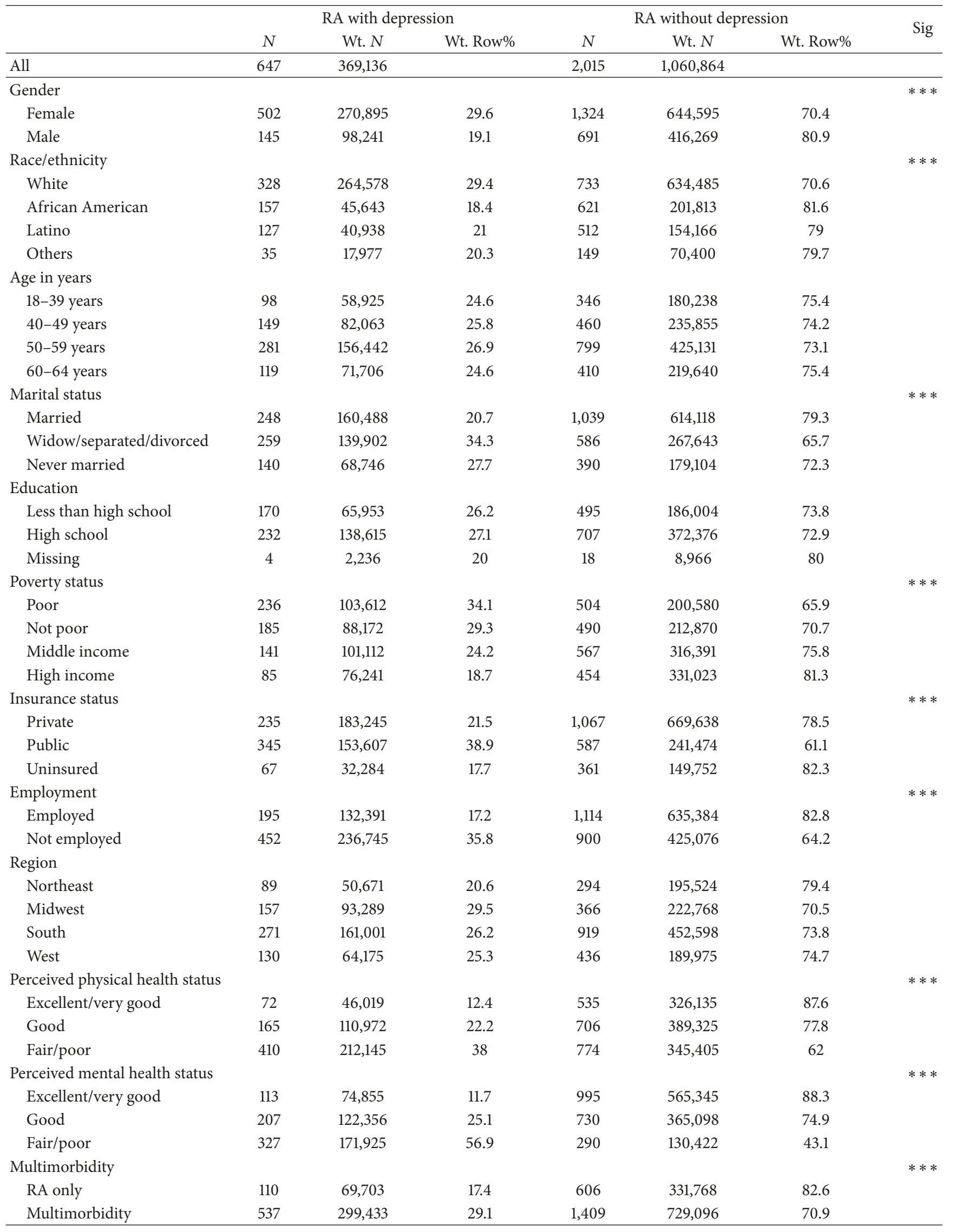


TABLE 2: Continued.

\begin{tabular}{|c|c|c|c|c|c|c|c|}
\hline & \multicolumn{3}{|c|}{ RA with depression } & \multicolumn{3}{|c|}{ RA without depression } & \multirow{2}{*}{ Sig } \\
\hline & $N$ & Wt. $N$ & Wt. Row\% & $N$ & Wt. $N$ & Wt. Row\% & \\
\hline Anxiety & & & & & & & $* * *$ \\
\hline Yes & 259 & 150,809 & 53.2 & 235 & 132,776 & 46.8 & \\
\hline No & 388 & 218,327 & 19 & 1,780 & 928,088 & 81 & \\
\hline Obesity & & & & & & & $* *$ \\
\hline Obese & 353 & 190,757 & 30.3 & 865 & 439,579 & 69.7 & \\
\hline Not obese & 287 & 172,382 & 22.4 & 1,105 & 598,794 & 77.6 & \\
\hline Smoking status & & & & & & & $* * *$ \\
\hline Current smoker & 250 & 142,876 & 36.5 & 448 & 248,988 & 63.5 & \\
\hline Others & 355 & 204,588 & 21.9 & 1,400 & 727,893 & 78.1 & \\
\hline Exercise & & & & & & & $* * *$ \\
\hline Yes & 190 & 106,306 & 18 & 887 & 484,895 & 82 & \\
\hline No exercise & 454 & 259,932 & 31.3 & 1,114 & 570,460 & 68.7 & \\
\hline
\end{tabular}

Note. Based on 2,662 adults with Rheumatoid Arthritis aged between 18 to 64 years, who were alive during the calendar year. Missing data for the variables, education, obesity, smoking, and exercise, are not presented. Asterisks represent significant group differences by the presence of depression based on chi-square tests. Weighted $N$ and percentages were derived by dividing the person weights by the number of years pooled; Wt.: weighted; ${ }^{* * *} p<.001 ; .001 \leq{ }^{* *} p<.01$.

TABLE 3: Clinical outcome associated with depression among working-age adults with Rheumatoid Arthritis Medical Expenditure Panel Survey $(2009,2011,2013$, and 2015).

\begin{tabular}{|c|c|c|c|c|c|}
\hline & \multicolumn{2}{|c|}{$\mathrm{RA}+$ depression } & \multicolumn{2}{|c|}{ RA and no depression } & \multirow{2}{*}{ Sig } \\
\hline & $N$ & $\begin{array}{c}\text { Wt. } \\
\text { col\% }\end{array}$ & $N$ & $\begin{array}{c}\text { Wt. } \\
\text { col\% }\end{array}$ & \\
\hline All & 605 & & 1,875 & & \\
\hline Pain interference with daily activity & & & & & $* * *$ \\
\hline Mild/none & 155 & 29.8 & 918 & 51.9 & \\
\hline Moderate & 92 & 15.4 & 317 & 17.3 & \\
\hline Severe (extreme/quite a lot) & 358 & 54.8 & 640 & 30.8 & \\
\hline \multicolumn{6}{|l|}{ Limitations } \\
\hline Any functional limitations & 528 & 79.0 & 1,026 & 51.1 & $* * *$ \\
\hline \multicolumn{6}{|c|}{ Adjusted odds ratio and 95\% CI for depression from multinomial logistic regression on pain interference with normal activity } \\
\hline & AOR & $95 \% \mathrm{CI}$ & & & Sig \\
\hline \multicolumn{6}{|l|}{ Pain interference with daily activity } \\
\hline \multicolumn{6}{|l|}{ Mild/none (reference group) } \\
\hline Moderate & 1.37 & {$[0.91,2.06]$} & & & \\
\hline Severe & 2.22 & {$[1.55,3.18]$} & & & $* * *$ \\
\hline \multicolumn{6}{|c|}{ Adjusted odds ratio and 95\% CI for depression from logistic regression on limitations } \\
\hline & AOR & $95 \%$ CI & & & Sig \\
\hline \multicolumn{6}{|l|}{ Limitations } \\
\hline Any functional limitations & 2.24 & {$[1.62,3.10]$} & & & $* * *$ \\
\hline
\end{tabular}

40.1\% adults without depression. Even after controlling for other factors mentioned in the methods section, adults with RA and depression were 1.55 times as likely as those without depression to be unemployed $(\mathrm{AOR}=1.55 ; 95 \% \mathrm{CI}=1.14$, 2.10). Among employed adults, those with depression had significantly higher number of missed work days annually (9 versus $6, p<.05)$ and higher lost wages ( $\$ 813$ versus $\$ 571$, $p<.05$ ) due to missed work (Table 5). We obtained similar results with counterfactual recycled predictions.

\section{Discussion}

In this study using a nationally representative sample of community-dwelling US adults, one in four working-age 
TABLE 4: Humanistic outcomes (health-related quality of measures) by presence of depression among working-age adults with Rheumatoid Arthritis Medical Expenditure Panel Survey (2009, 2011, 2013, and 2015).

\begin{tabular}{|c|c|c|c|c|c|}
\hline & \multicolumn{2}{|c|}{ RA + depression } & \multicolumn{2}{|c|}{ RA and no depression } & \multirow{2}{*}{ Sig } \\
\hline & Wt. mean & SE & Wt. mean & SE & \\
\hline All & $N=647$ & & $N=2,015$ & & \\
\hline Physical component summary score & 35.07 & 0.89 & 40.18 & 0.47 & $* * *$ \\
\hline $\begin{array}{l}\text { Mental component summary } \\
\text { score }\end{array}$ & 37.20 & 0.77 & 48.74 & 0.37 & $* * *$ \\
\hline
\end{tabular}

Fully adjusted model: parameter estimates and standard errors for depression ordinary least squares regression mental component summary score

\begin{tabular}{|c|c|c|c|}
\hline & Beta & Standard error & Sig \\
\hline Depression & -8.72 & 0.81 & $* * *$ \\
\hline \multicolumn{4}{|l|}{ No depression (reference group) } \\
\hline \multicolumn{4}{|c|}{$\begin{array}{l}\text { Fully adjusted model: parameter estimates and standard errors for depression ordinary least squares regression physical } \\
\text { component summary score }\end{array}$} \\
\hline & Beta & Standard error & Sig \\
\hline Depression & -1.29 & 0.81 & \\
\hline No depression (reference group) & & & \\
\hline
\end{tabular}

Note. Based on 2,662 adults with Rheumatoid Arthritis aged between 18 and 64 years, who were alive during the calendar year. Asterisks represent significant group differences by the presence of depression. The ordinary least squares regressions controlled for the following variables: sex, race/ethnicity, age, region, marital status, education, family poverty status, health insurance, anxiety, multimorbidity, obesity, physical activity, and smoking; SE: standard error; Wt.: weighted; ${ }^{* * *} p<.001$.

adults with RA reported depression. This rate is considerably higher compared to the $6.8 \%$ rate of depression in the general population in the US [27] and higher than the pooled depression rate of $16.8 \%$ reported by Matcham and colleagues in a meta-analysis of 72 studies that included 13,189 RA patients [5]. The same meta-analysis also reported the presence of depressive symptoms in $38.8 \%$ of RA patients measured using Patient Health Questionnaire (PHQ-9) and $34.2 \%$ of RA patients measured using Hospital Anxiety and Depression Scale (HADS) [5]. Therefore, the differences in the rate of depression in RA patients can be explained by the differences in the instruments used to identify depression.

Our study findings indicated the substantial additional clinical burden imposed by depression in working-age adults with RA. These findings have implications for comanagement of depression and RA. Although not specific to RA, a randomized clinical trial of 1,001 patients with concurrent depression and arthritis and seeking care from 18 primary care clinics [28] suggested that collaborative depression care not only reduced depressive symptoms but also improved arthritis related outcomes, such as decreasing pain and functional limitations. There is some evidence that diseasemodifying drugs used to treat RA can have spill-over effects in reducing depressive symptoms. For example, depression levels decreased significantly following commencement and continuity of rituximab, a B cell-directed therapy, among individuals with RA [29]. Therefore, future studies need to systematically evaluate whether antirheumatic treatment among individuals with RA can help alleviate depressive symptoms.

We also observed significant decrements in HRQoL measures, specifically the MCS scores. This is not surprising; however, it is important given the strong association between patient-reported outcomes and disease activity [30]. It has also been suggested that patient-reported outcomes such as the HRQoL and other measures in clinical trials and routine clinical practice may shed light on variations in treatment response as well as the burden of disease among RA adults $[30,31]$. Our findings suggest that collecting patient-reported HRQoL can be critical in assessing disease burden that may not be captured by clinical assessment alone [30].

Depression in working-age adults with RA was associated with substantial direct and indirect economic burden. For example, the presence of depression more than doubled the annual per person total healthcare costs, a number of missed work days, and lost wages due to missed work days, even after controlling for predisposing factors, enabling characteristics, need factors, and personal health care practices. Although published evidence on the incremental economic impact of depression in RA is limited [9], our findings which are consistent with studies assessing the burden of depression on other chronic illnesses such as diabetes, cardiovascular disease, and asthma have also reported the synergistic effect of depression in increasing the economic burden among individuals with chronic conditions $[12,32]$.

Our findings on the economic burden of depression in RA patients have important implications for the payers as it highlights an opportunity for reducing expenditures in RA patients by increasing efforts towards screening and effectively treating depression in RA patients. Potential strategies could be improving the integration of mental health services with rheumatology practice and facilitating mental health training for rheumatologists. Future studies need to explore whether treatment for depression provides an opportunity to reduce direct healthcare expenditures associated with depression in RA patients. 
TABLE 5: Economic outcomes by presence of depression among working-age adults with Rheumatoid Arthritis Medical Expenditure Panel Survey $(2009,2011,2013$, and 2015).

\begin{tabular}{|c|c|c|c|c|c|}
\hline & \multicolumn{2}{|c|}{$\mathrm{RA}+$ depression } & \multicolumn{2}{|c|}{ RA and no depression } & \multirow{2}{*}{ Sig } \\
\hline & Wt. mean & SE & Wt. mean & SE & \\
\hline All & $N=647$ & & $N=2,015$ & & \\
\hline Total healthcare expenditures (2015 \$) & 17,941 & 1489 & 10,064 & 574 & $* * *$ \\
\hline $\begin{array}{l}\text { Total out-of-pocket spending by } \\
\text { patients/families }(2015 \$)\end{array}$ & $\$ 1,443$ & 135 & $\$ 1,052$ & 73 & $* * *$ \\
\hline \multicolumn{6}{|c|}{ Adjusted total direct healthcare expenditures of depression from generalized linear models with gamma distribution and log link } \\
\hline & Wt. mean & $95 \% \mathrm{CI}$ & Wt. mean & $95 \% \mathrm{CI}$ & Sig \\
\hline Total healthcare expenditures (2015 \$) & 14,752 & $(14,411-15,125)$ & 10,541 & $(10,206-10,806)$ & $* * *$ \\
\hline $\begin{array}{l}\text { Total out-of-pocket spending by } \\
\text { patients/families }(2015 \$)\end{array}$ & 1,232 & $(1,202-1265)$ & 979 & $(955-1,005)$ & $* * *$ \\
\hline
\end{tabular}
patients/families $(2015 \$)$

Incremental total direct healthcare expenditures of depression from counterfactual recycled prediction

\begin{tabular}{|c|c|c|c|}
\hline & Wt. mean & $95 \%$ CI & \\
\hline Total healthcare expenditures (2015 \$) & 4,212 & $(4,114,4318)$ & $* * *$ \\
\hline $\begin{array}{l}\text { Total out-of-pocket spending by } \\
\text { patients/their families }(2015 \$)\end{array}$ & 253 & $(247-260)$ & $* * *$ \\
\hline
\end{tabular}

High out-of-pocket spending burden (>10\% income spent on healthcare)

\begin{tabular}{|c|c|c|c|c|c|}
\hline & \multicolumn{2}{|c|}{$\mathrm{RA}+$ depression } & \multicolumn{3}{|c|}{ RA and no depression } \\
\hline & $N$ & Wt. col\% & $N$ & Wt. col\% & \\
\hline High out-of-pocket spending burden & 192 & 30.7 & 424 & 21.3 & $* * *$ \\
\hline \multicolumn{6}{|c|}{$\begin{array}{c}\text { Fully adjusted model: adjusted odds ratio (AOR) and 95\% confidence intervals (CI) of Depression from logistic regression on high } \\
\text { out-of-pocket burden }\end{array}$} \\
\hline & AOR & $95 \% \mathrm{CI}$ & Sig & & \\
\hline Depression & 1.34 & {$[1.01,1.79]$} & & & $* *$ \\
\hline No depression (reference) & & & & & \\
\hline
\end{tabular}

Unemployment among working-age adults

\begin{tabular}{|c|c|c|c|c|c|}
\hline & \multicolumn{2}{|c|}{$\mathrm{RA}+$ depression } & \multicolumn{3}{|c|}{ RA and no depression } \\
\hline & $N$ & Wt. col\% & $N$ & Wt. col\% & \\
\hline Unemployed & 452 & 64.1 & 900 & 40.1 & $* * *$ \\
\hline \multicolumn{6}{|c|}{$\begin{array}{c}\text { Fully adjusted model: adjusted odds ratio (AOR) and 95\% confidence intervals (CI) of depression from logistic regression on } \\
\text { unemployment }\end{array}$} \\
\hline & AOR & $95 \% \mathrm{CI}$ & Sig & & \\
\hline Depression & 1.55 & {$[1.14,2.10]$} & $* * *$ & & \\
\hline No depression (reference) & & & & & \\
\hline
\end{tabular}

Fully adjusted models: total productivity losses by depression from negative binomial regression on missed work Days

Wt. mean $\quad 95 \% \mathrm{CI} \quad$ Wt. mean $95 \% \mathrm{CI}$

Sig

\begin{tabular}{|c|c|c|c|c|c|}
\hline Number of missed work days & 9.0 & $(8.7-9.4)$ & 6.0 & $(5.7-6.2)$ & \\
\hline Lost wages & 853 & $(833-873)$ & 571 & $(558-584)$ & $* * *$ \\
\hline
\end{tabular}

Incremental total productivity losses associated with depression from counterfactual recycled prediction

Wt. mean $\quad 95 \%$ CI

\begin{tabular}{lcc}
\hline Number of missed work days & 3.1 & $(2.9-3.2)$ \\
Lost wages & 282 & $(276-289)$ \\
\hline
\end{tabular}

Note. Based on 2,662 adults with Rheumatoid Arthritis aged between 18 and 64 years, who were alive during the calendar year. Asterisks represent significant group differences by the presence of depression. The adjusted models squares regressions controlled for the following variables: sex, race/ethnicity, age, region, marital status, education, family poverty status, health insurance, physical health, mental health status, anxiety, multimorbidity, obesity, physical activity, and smoking. Missed work days and lost wages were estimated only for those who were employed; ${ }^{* * *} p<.001 ; .001 \leq{ }^{* *} p<.01 ; .01 \leq{ }^{*} p<.05$. 
The study findings have important implications for the employers because depression costs US employers more than $\$ 31$ billion annually due to missed work and decreased work performance [33]. One study done in the US reported that depression leads to the highest reduction in work performance and the highest employer burden relative to any other chronic conditions [34]. Strategies that employers may adopt to improve mental health in employees include organizing workplace health promotion programs and stress management projects, which have shown the benefits of prevention and management of depression in workplace [35, 36].

To the best of our knowledge, this is the first populationbased study that comprehensively examined the excess clinical, humanistic, and economic burden of depression in working-age adults with RA. Other strengths of this study include the use of nationally representative survey, adjustment of a comprehensive list of confounders such as predisposing factors, enabling factors, need factors, and personal health care practices and the use of robust statistical techniques such as GLM, and recycled prediction in estimating the incremental costs and missed workdays.

However, the findings of this study should be interpreted considering its potential limitations. First, we did not control for the severity and duration of RA and depression as MEPS does not contain this information. These factors can be important confounders of both healthcare costs and work absence. Second, we have measured productivity loss as missed work days and did not consider other kinds of productivity loss such as reduced productivity while at work (presenteeism) and loss of employment.

Our findings would provide valuable insights to payers and other decision-makers to better understand the economic impact of comorbid depression on working RA patients from US societal perspective. It is well-documented that depression in RA patients is often underrecognized and undertreated in routine clinical practice $[37,38]$. Therefore, our study underscores the need for incorporating depression screening and management in the routine clinical management of RA in order to offset the substantial incremental costs associated with depression. Published evidence has well documented that depression is a treatable condition. However, it is still not clear whether depression treatment is equally effective in RA patients as compared to those with depression without RA [39]. Future studies need to assess the potential cost reductions that can be achieved through early detection and more aggressive treatment of depression in RA patients.

\section{Disclosure}

The content is solely the responsibility of the authors and does not necessarily represent the views/opinions of any organization.

\section{Conflicts of Interest}

The authors declare that they have no conflicts of interest.

\section{Acknowledgments}

The project was supported by the National Institute of General Medical Sciences, 2U54GM104942.

\section{References}

[1] World Health Organization, Chronic rheumatic conditions, World Health Organization, 2017, http://www.who.int/chp/ topics/rheumatic/en/.

[2] S. Allaire, F. Wolfe, J. Niu, and M. P. Lavalley, "Contemporary prevalence and incidence of work disability associated with rheumatoid arthritis in the US," Arthritis Care \& Research, vol. 59, no. 4, pp. 474-480, 2008.

[3] C. Dickens, L. McGowan, D. Clark-Carter, and F. Creed, "Depression in rheumatoid arthritis: a systematic review of the literature with meta-analysis," Psychosomatic Medicine, vol. 64, no. 1, pp. 52-60, 2002.

[4] U. Sambamoorthi, D. Shah, and X. Zhao, "Healthcare burden of depression in adults with arthritis," Expert Review of Pharmacoeconomics \& Outcomes Research, vol. 17, no. 1, pp. 53-65, 2017.

[5] F. Matcham, L. Rayner, S. Steer, and M. Hotopf, “The prevalence of depression in rheumatoid arthritis: A systematic review and meta-analysis," Rheumatology, vol. 52, no. 12, Article ID ket169, pp. 2136-2148, 2013.

[6] D. C. Ang, H. Choi, K. Kroenke, and F. Wolfe, "Comorbid depression is an independent risk factor for mortality in patients with rheumatoid arthritis," The Journal of Rheumatology, vol. 32, no. 6, pp. 1013-1019, 2005.

[7] F. Matcham, S. Norton, D. L. Scott, S. Steer, and M. Hotopf, "Symptoms of depression and anxiety predict treatment response and long-term physical health outcomes in rheumatoid arthritis: Secondary analysis of a randomized controlled trial," Rheumatology, vol. 55, no. 2, pp. 268-278, 2015.

[8] M. Kojima, T. Kojima, S. Suzuki et al., "Depression, inflammation, and pain in patients with rheumatoid arthritis," Arthritis Care \& Research, vol. 61, no. 8, pp. 1018-1024, 2009.

[9] A. T. Joyce, P. Smith, R. Khandker, J. M. Melin, and A. Singh, "Hidden cost of rheumatoid arthritis (RA): Estimating cost of comorbid cardiovascular disease and depression among patients with RA," The Journal of Rheumatology, vol. 36, no. 4, pp. 743-752, 2009.

[10] P. P. Katz and E. H. Yelin, "Prevalence and correlates of depressive symptoms among persons with rheumatoid arthritis," The Journal of Rheumatology, vol. 20, no. 5, pp. 790-796, 1993.

[11] K. A. Theis, L. Murphy, J. M. Hootman, C. G. Helmick, and E. Yelin, "Prevalence and correlates of arthritis-attributable work limitation in the US population among persons ages 18-64: 2002 National health interview survey data," Arthritis Care \& Research, vol. 57, no. 3, pp. 355-363, 2007.

[12] M. B. Stein, B. J. Cox, T. O. Afifi, S.-L. Belik, and J. Sareen, "Does co-morbid depressive illness magnify the impact of chronic physical illness? A population-based perspective," Psychological Medicine, vol. 36, no. 5, pp. 587-596, 2006.

[13] D. C. Uhm, E. S. Nam, H. Y. Lee, E. B. Lee, Y. I. Yoon, and G. J. Chai, "Health-related quality of life in korean patients with rheumatoid arthritis: association with pain, disease activity, disability in activities of daily living and depression," Journal of Korean Academy of Nursing, vol. 42, no. 3, p. 434, 2012.

[14] E. M. Abu Al-Fadl, M. A. Ismail, M. Thabit, and Y. ElSerogy, "Assessment of health-related quality of life, anxiety and 
depression in patients with early rheumatoid arthritis," Egyptian Rheumatologist, vol. 36, no. 2, pp. 51-56, 2014.

[15] T. Mikuls, "Health related quality of life in women with elderly onset rheumatoid arthritis," The Journal of Rheumatology, vol. 30, no. 5, p. 952, 2003.

[16] N. Joshi, R. Khanna, and R. M. Shah, "Relationship between depression and physical activity, disability, burden, and healthrelated quality of life among patients with arthritis," Population Health Management, vol. 18, no. 2, pp. 104-114, 2015.

[17] J. P. Sommers, An examination of state estimates using multiple years of data from the medical expenditure panel survey, household component, Agency for Healthcare Research and Quality, 2006.

[18] R. M. Andersen, "Revisiting the behavioral model and access to medical care: does it matter?" Journal of Health and Social Behavior, vol. 36, no. 1, pp. 1-10, 1995.

[19] D. J. Gaskin and P. Richard, "The economic costs of pain in the United States," The Journal of Pain, vol. 13, no. 8, pp. 715-724, 2012.

[20] J. E. Ware Jr., M. Kosinski, and S. D. Keller, "A 12-Item shortform health survey: construction of scales and preliminary tests of reliability and validity," Medical Care, vol. 34, no. 3, pp. 220233, 1996.

[21] S. Crystal et al., "Out-of-pocket health care costs among older Americans," The Journals of Gerontology Series B: Psychological Sciences and Social Sciences, vol. 55, no. 1, pp. S51-S62, 2000.

[22] K. A. Paez, L. Zhao, and W. Hwang, "Rising out-of-pocket spending for chronic conditions: A ten-year trend," Health Affairs, vol. 28, no. 1, pp. 15-25, 2009.

[23] A. Deb, U. Sambamoorthi, J. D. Thornton, B. Schreurs, and K. Innes, "Direct medical expenditures associated with Alzheimer's and related dementias (ADRD) in a nationally representative sample of older adults - an excess cost approach," Aging \& Mental Health, pp. 1-6, 2017.

[24] A. Basu and P. J. Rathouz, "Estimating marginal and incremental effects on health outcomes using flexible link and variance function models," Biostatistics, vol. 6, no. 1, pp. 93-109, 2005.

[25] AHRQ, MEPS HC-036: 1996-2007 Pooled Estimation File, 2009, https://meps.ahrq.gov/data_stats/download_data/pufs/h36/ h36u07doc.shtml.

[26] D. Coughlan, S. T. Yeh, C. O’Neill, and K. D. Frick, "Evaluating direct medical expenditures estimation methods of adults using the medical expenditure panel survey: An example focusing on head and neck cancer," Value in Health, vol. 17, no. 1, pp. 90-97, 2014.

[27] CDC, "Current depression among adults-United States, 2006 and 2008," MMWR: Morbidity and mortality weekly report 38, 2010.

[28] E. B. Lin et al., "Effect of improving depression care on pain and functional outcomes among older adults with arthritis: A randomized controlled trial," JAMA, vol. 290, no. 18, pp. 24282429, 2003.

[29] I. R. Gaisin and L. V. Ivanova, "FRI0239 Decreased prevalence of anxiety and depression in rheumatoid arthritis patients following b cell-targeted biologic therapy," Annals of the Rheumatic Diseases, vol. 72, no. Suppl 3, pp. A454.2-A454, 2014.

[30] A.-M. Orbai and C. O. Bingham, "Patient Reported Outcomes in Rheumatoid Arthritis Clinical Trials," Current Rheumatology Reports, vol. 17, no. 4, 2015.

[31] M. M. Ward, L. C. Guthrie, and M. I. Alba, "Rheumatoid arthritis response criteria and patient-reported improvement in arthritis activity: Is an American College of Rheumatology twenty percent response meaningful to patients?" Arthritis \& Rheumatology, vol. 66, no. 9, pp. 2339-2343, 2014.

[32] L. E. Egede, K. G. Bishu, R. J. Walker, and C. E. Dismuke, "Impact of diagnosed depression on healthcare costs in adults with and without diabetes: United States, 2004-2011," Journal of Affective Disorders, vol. 195, pp. 119-126, 2016.

[33] W. F. Stewart, J. A. Ricci, E. Chee, S. R. Hahn, and D. Morganstein, "Cost of lost productive work time among US workers with depression," The Journal of the American Medical Association, vol. 289, no. 23, pp. 3135-3144, 2003.

[34] R. Kessler, L. A. White, H. Birnbaum et al., "Comparative and interactive effects of depression relative to other health problems on work performance in the workforce of a large employer," Journal of Occupational and Environmental Medicine, vol. 50, no. 7, pp. 809-816, 2008.

[35] A. D. LaMontagne, A. Martin, K. M. Page et al., "Workplace mental health: developing an integrated intervention approach," BMC Psychiatry, vol. 14, article 131, 2014.

[36] D. McDaid and A.-L. Park, "Investing in mental health and wellbeing: Findings from the DataPrev project," Health Promotion International, vol. 26, no. 1, pp. i108-i139, 2011.

[37] M. Margaretten, L. Julian, P. Katz, and E. Yelin, "Depression in patients with rheumatoid arthritis: Description, causes and mechanisms," International Journal of Clinical Rheumatology, vol. 6, no. 6, pp. 617-623, 2011.

[38] S. L. Hider, W. Tanveer, A. Brownfield, D. L. Mattey, and J. C. Packham, "Depression in RA patients treated with anti-TNF is common and under-recognized in the rheumatology clinic," Rheumatology, vol. 48, no. 9, pp. 1152-1154, 2009.

[39] K. M. Fiest, C. A. Hitchon, C. N. Bernstein et al., "Systematic review and meta-analysis of interventions for depression and anxiety in persons with rheumatoid arthritis," JCR: Journal of Clinical Rheumatology, vol. 23, no. 8, pp. 425-434, 2017. 


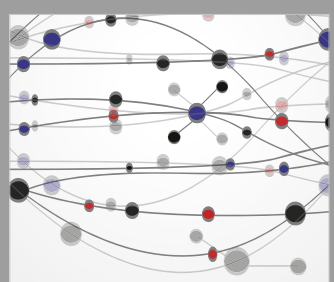

The Scientific World Journal
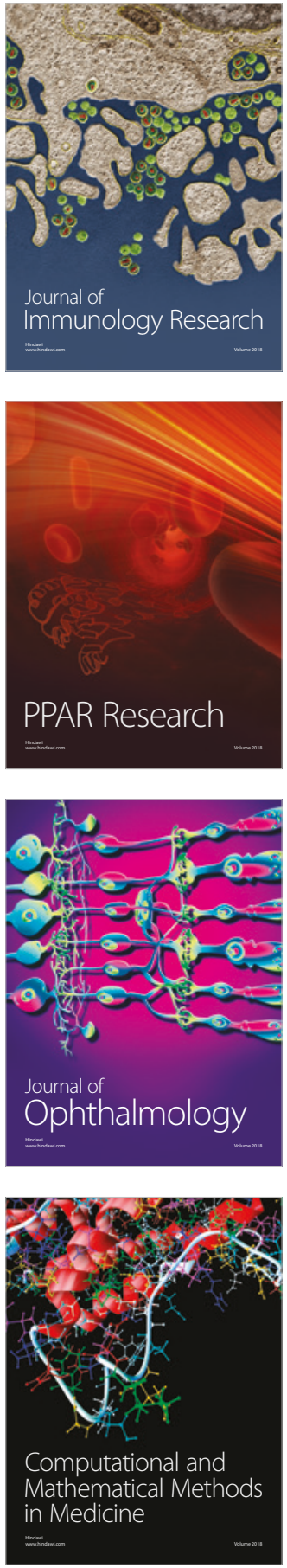

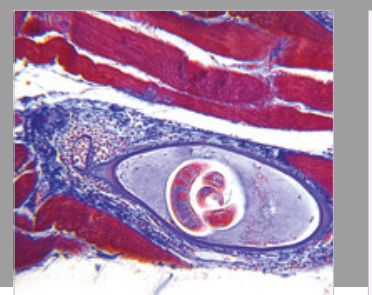

Gastroenterology Research and Practice

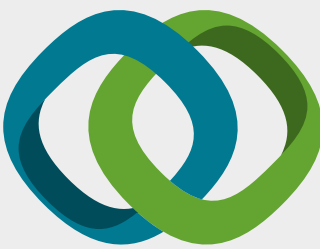

\section{Hindawi}

Submit your manuscripts at

www.hindawi.com
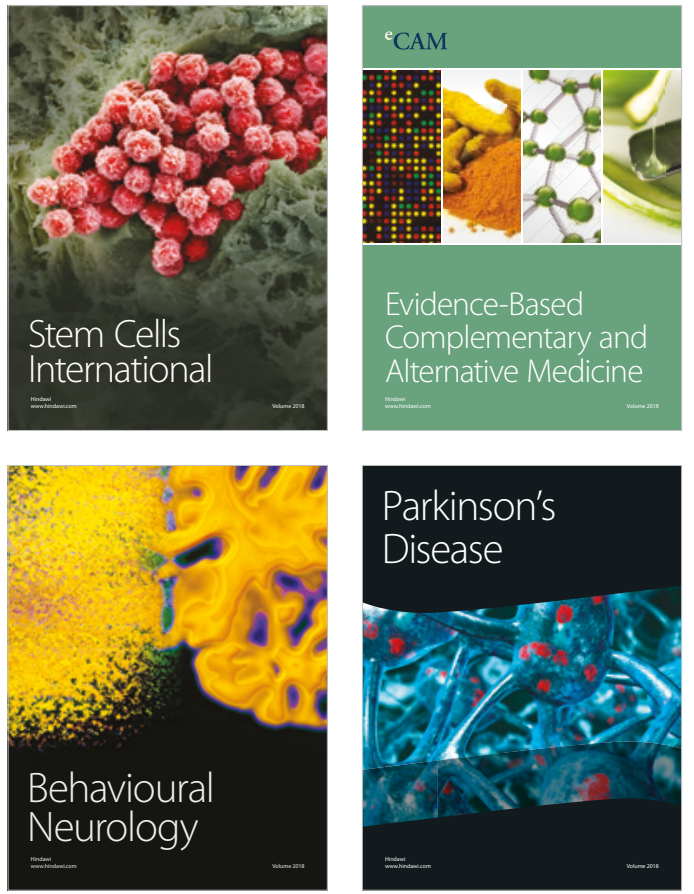

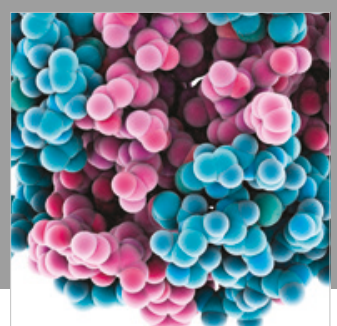

ournal of

Diabetes Research

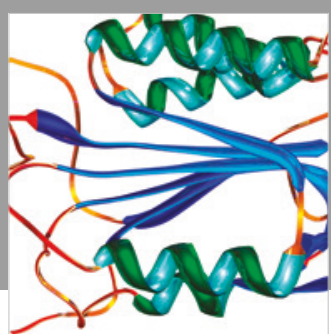

Disease Markers
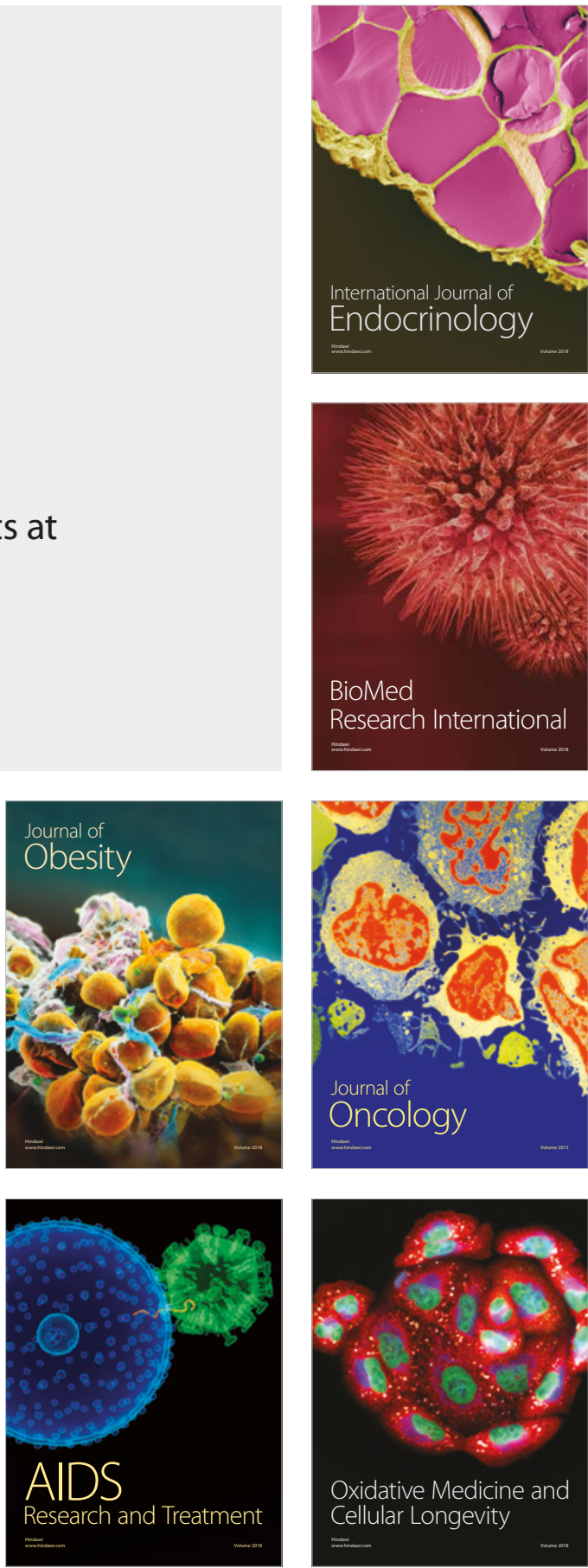